\title{
Weakly hydrated anions bind to polymers but not monomers in aqueous solutions
}

\author{
Bradley A. Rogers ${ }^{1}$, Halil I. Okur ${ }^{1,4}$, Chuanyu Yan', Tinglu Yang1, Jan Heyda $\mathbb{1}^{2}$ and Paul S. Cremer ${ }^{1,3 凶}$ \\ Weakly hydrated anions help to solubilize hydrophobic macromolecules in aqueous solutions, but small molecules comprising \\ the same chemical constituents precipitate out when exposed to these ions. Here, this apparent contradiction is resolved by \\ systematically investigating the interactions of NaSCN with polyethylene oxide oligomers and polymers of varying molecular \\ weight. A combination of spectroscopic and computational results reveals that $\mathbf{S C N}^{-}$accumulates near the surface of polymers, \\ but is excluded from monomers. This occurs because $\mathbf{S C N}^{-}$preferentially binds to the centre of macromolecular chains, where \\ the local water hydrogen-bonding network is disrupted. These findings suggest a link between ion-specific effects and theories \\ addressing how hydrophobic hydration is modulated by the size and shape of a hydrophobic entity.
}

W eakly hydrated anions, such as $\mathrm{I}^{-}, \mathrm{SCN}^{-}$and $\mathrm{ClO}_{4}^{-}$, weaken the hydrophobic effect in aqueous solutions. These large, polarizable anions denature proteins, inhibit supramolecular complexation and dissolve surfactant micelles ${ }^{1-3}$. At the molecular level, weakly hydrated anions partially shed their hydration shells and adsorb to nonpolar interfaces, thereby inhibiting hydrophobic assembly ${ }^{4-10}$. The adsorption of these anions to amide-rich polymers has been characterized by submolar to molar equilibrium dissociation constants, $K_{\mathrm{D}}=0.05-1.60 \mathrm{M}$ (refs. ${ }^{11-14}$ ). Even tighter adsorption has been observed at macroscopic surfaces, such as the air/water interface $\left(K_{\mathrm{D}}=0.03-0.26 \mathrm{M}\right)$ and in the concave pockets of cavitands and proteins $\left(K_{\mathrm{D}}=0.003-0.09 \mathrm{M}\right)^{2,15-22}$. By stark contrast, anions are repelled from small molecules, like $N$-methyl acetamide and tert-butyl alcohol $\left(K_{\mathrm{D}}=4-8 \mathrm{M}\right)^{23,24}$. As a consequence, weakly hydrated anions precipitate small non-ionic solutes out of aqueous solutions, including acetone and diacetone alcohol $^{25}$. The dramatic range of anion affinity for chemically similar aliphatic binding sites exposes a critical gap in our knowledge of the mechanisms for anion-specific effects.

The surface curvature of nonpolar solutes is known to influence solubility because of the distinct local hydration of curved and flat interfaces ${ }^{26,27}$. The water hydrogen-bonding network can wrap around small and convex solutes to maintain its bulk-like structure. Large solutes, however, have a flatter topography that disrupts the hydrogen bonds between water molecules ${ }^{27}$. As such, small solutes can be incorporated into the water network, while larger ones with broken hydrogen bonds associate with each other and release water molecules into the bulk solution. The cartoon in Fig. 1 depicts a simple model for a polymer chain. The termini are highly curved due to their half-spherical geometry, while the centre of the chain is flatter because it has a cylindrical-like structure. Experimental and computational studies of nonpolar solutes with varying chain lengths have shown that this topography disorders water at the centre of the chain more than at the termini ${ }^{28,29}$.

Here, the role of surface curvature on anion-specific effects is explored by systematically measuring the interactions of NaSCN with polyethylene oxides (PEO) of varying molecular weights, ranging from monomers to polymers. The results indicate that $\mathrm{SCN}^{-}$is repelled from monomers but attracted to oligomers of increasing chain length. These interactions are distinct because $\mathrm{SCN}^{-}$binds selectively to the centre of oligomer chains, as opposed to their termini (Fig. 1). Investigations of polyether hydration shells reveal that the water structure at the centre of the chain is more disordered than at the termini. Together, these findings imply that $\mathrm{SCN}^{-}$interacts with low-curvature interfaces to displace water at sites of hydrogen-bonding defects. The correlation of binding affinity and water structure measurements at specific locations along the polyether solutes establishes a link between ion-specific effects (Hofmeister series chemistry) and the hydration of hydrophobic interfaces.

\section{Results}

Heterogeneous adsorption of weakly hydrated anions to polyether chains. The adsorption of $\mathrm{NaSCN}$ to pentaethylene oxide (PEO-5) was monitored by ${ }^{1} \mathrm{H}$ NMR spectroscopy. The nature of the interaction was determined from changes in the solute's proton chemical shifts, $\Delta \delta$, at a given salt concentration, $c_{\text {salt }}$ as shown in equation (1):

$$
\Delta \delta=a c_{\text {salt }}+\frac{\delta_{\text {max }} c_{\text {salt }}}{K_{\mathrm{D}}+c_{\text {salt }}}
$$

The four spectrally unique proton positions for PEO-5 are denoted 'central', 'penultimate', 'end' and 'termini' in Fig. 2a. The chemical shift values associated with the terminal methyl protons (blue data points) and internal methylene protons (red, orange and green data points) decrease following the introduction of NaSCN (Fig. 2b). The decrease at the termini is linear, with a slope value of $a$, as modelled by the first term in equation (1). By contrast, data from the internal methylene groups displays nonlinear behaviour below $0.1 \mathrm{M} \mathrm{NaSCN}$. The nonlinearity can be fit to a Langmuir binding isotherm, as defined by the second term in equation (1). This nonlinear term corresponds to the small, saturable increase in the chemical shift, $\delta_{\max }$, as $\mathrm{SCN}^{-}$binds to the

'Department of Chemistry, The Pennsylvania State University, University Park, PA, USA. ${ }^{2}$ Department of Physical Chemistry, University of Chemistry and Technology, Prague, Dejvice, Czech Republic. ${ }^{3}$ Department of Biochemistry and Molecular Biology, The Pennsylvania State University, University Park, PA, USA. ${ }^{4}$ Present address: Department of Chemistry and National Nanotechnology Research Center (UNAM), Bilkent University, Ankara, Turkey. 凶e-mail: psc11@psu.edu 


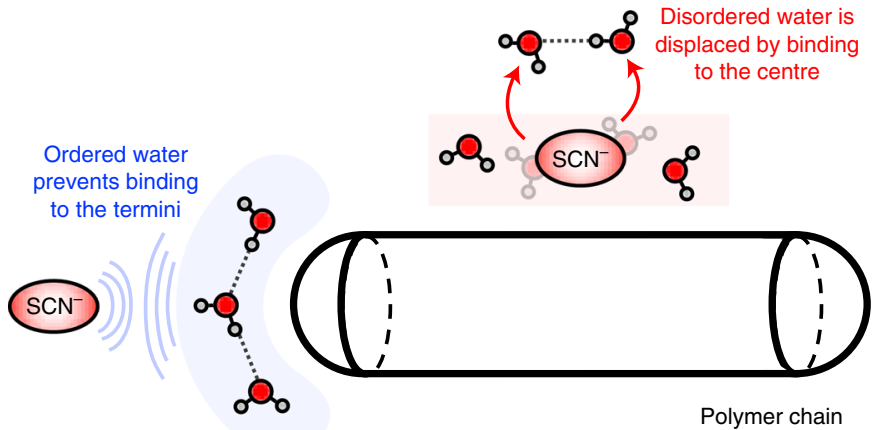

Fig. 1 | Schematic of a polymer chain and the effects its surface curvature has on interfacial water structure and $\mathrm{SCN}^{-}$adsorption. The hydration

shell is well ordered near the curved termini, which leads to the exclusion of $\mathrm{SCN}^{-}$(blue region). By contrast, $\mathrm{SCN}^{-}$readily adsorbs to the centre of the chain because the water is more disordered by its flatter surface curvature (red region).

chain. The binding strength at the internal methylene groups can be quantified by a submolar equilibrium dissociation constant, $K_{\mathrm{D}}=0.1 \mathrm{M}$. The value of $K_{\mathrm{D}}$ at the termini, however, is too weak to be detected, $K_{\mathrm{D}}>2.4 \mathrm{M}$.

The free energy of adsorption, that is, $\Delta G_{\text {ads }}=R T \ln \left(K_{\mathrm{D}}\right)$, is plotted for specific positions on the PEO-5 chain in Fig. $2 \mathrm{c}$ (unfilled data points), where $R$ is the ideal gas constant and $T$ is the temperature. Positive values of $\Delta G_{\text {ads }}$ indicate repulsion of $\mathrm{NaSCN}$, while negative values denote attraction. As can be seen, $\mathrm{SCN}^{-}$was repelled from the methyl termini, but attracted to the methylenes along the interior of the chain. Figure $2 \mathrm{c}$ also includes the average values for $\Delta G_{\text {ads }}$ (filled data points) obtained from analogous NMR experiments that were performed on a large set of polyethers with different chain lengths. The chain lengths ranged from the monomer to a 20,000-mer, as illustrated in Fig. 2d. These results indicate that salt ions were repelled from the methyl termini and attracted to the centre of the chain, regardless of chain length.

All-atom molecular dynamics (MD) simulations of PEO-5 in NaSCN solutions also revealed heterogeneous adsorption of the salt ions. The interaction between the ions and the solute was quantified by the preferential interaction coefficient, $\Gamma_{23}$, where water, solute and salt are designated by the indices 1, 2 and 3, respectively ${ }^{30}$. Positive values of $\Gamma_{23}$ represent an accumulation of NaSCN relative to the bulk concentration, whereas negative values denote depletion. The values of $\Gamma_{23}$ were normalized to both the salt concentration and the solvent accessible surface area (SASA) and referred to as $\mu_{23}$ (ref. $\left.{ }^{31}\right)$. The $\mu_{23}$ data are organized by position along the PEO-5 chain in Fig. 2e (unfilled data points). The average values of $\mu_{23}$, which were obtained from MD simulations of different polyethers of varying chain length, are also provided in Fig. 2e (filled data points). As can be seen, salt ions accumulate near the internal aliphatic moieties and are slightly depleted from the terminal methyl groups.

Collectively, these results suggest that the adsorption of $\mathrm{SCN}^{-}$ to the polyether chain is heterogeneous, with tighter binding at the centre of the chain and weaker interactions near the termini. This is the case, despite the fact that the monomer chemistry is essentially identical at each position. Such findings imply that the tighter affinity to long chains occurs because the concentration of the termini is diluted with increasing chain length. Moreover, these studies are consistent with a surface curvature hypothesis. Namely, the binding affinity appears to correlate with the lower curvature at the cylindrical centre of the chains, as opposed to the half-spherical terminal segments, which exhibit higher curvature (Fig. 1).
Structure of water in polyether hydration shells. The mechanism depicted in Fig. 1 suggests that the adsorption of weakly hydrated anions to polymer interfaces may be controlled by the differences in hydration of the centre and termini moieties of the chain. As such, Raman-multivariate curve resolution (Raman-MCR) spectroscopy was employed to probe the structure of the polyether hydration shells in neat water ${ }^{32}$. MCR analysis allows the Raman spectrum of a solution to be separated into two components that correspond to the solute hydration shell and the bulk water, as depicted schematically in Fig. 3a. The distinction between these two regions is illustrated by the dashed black outline around the polymer chain. The hydration shell spectra of PEO-1 and PEO-2,000 are shown in Fig. 3b. Both spectra display a broad $\mathrm{OH}$ stretch peak between 3,000 and $3,600 \mathrm{~cm}^{-1}$ that reports on water molecules in the hydration shell. The solid blue and red lines in Fig. $3 \mathrm{~b}$ are fits to the data using two Gaussian peaks for the $\mathrm{OH}$ stretch region. The peak at $3,250 \mathrm{~cm}^{-1}$ (vertical dashed blue line) can be assigned to more tetrahedrally ordered water molecules, and the peak near $3,450 \mathrm{~cm}^{-1}$ (vertical dashed red line) is attributed to water molecules with weaker and less tetrahedral hydrogen bonds $\mathrm{s}^{33-37}$.

The area ratio of the $3,250 \mathrm{~cm}^{-1}$ peak to the $3,450 \mathrm{~cm}^{-1}$ peak, $A_{3,250} / A_{3,450}$, can be used as a metric for the structure of water around a given chain, with higher values corresponding to increased ordering. Raman-MCR measurements for the entire set of polyethers reveal a chain length dependence to the $A_{3,250} / A_{3,450}$ value (Fig. $3 \mathrm{c}$ ). As can be seen, the value of $A_{3,250} / A_{3,450}$ decreases with chain length, implying a disruption of the tetrahedral water structure at the centre of the chain. Indeed, the hydration shells of longer chains should be dominated by water molecules solvating central groups, rather than the termini, as they constitute most of the SASA. The black curve in Fig. $3 \mathrm{c}$ is a fit to the data using a segmented chain model, where the total value of $A_{3,250} / A_{3,450}$ is calculated as the linear combination of the segment-specific ratios, $\left(A_{3,250} / A_{3,450}\right)_{i}$, that are weighted by their fraction of the SASA. Four segments were used in the fit based on the binding sites observed in the NMR experiments (Fig. 2).

The structure of water in the MD simulations can be quantified by the tetrahedral order parameter, $q$ (ref. ${ }^{38}$ ). Water molecules in ideal tetrahedral hydrogen-bonding geometries yield a $q$ value of 1 , while random geometries, akin to an ideal gas, give rise to $q=0$. The probability distributions of the tetrahedral order parameter, $P(q)$, for the terminal (blue) and centre (red) segments of the PEO-5 hydration shell as well as bulk water (black) are plotted in Fig. 3d. As can be seen, the probability of observing more tetrahedral hydrogen bonding, $P(0.76)$, is higher in bulk water than in the vicinity of the methyl termini (vertical dashed blue line). Moreover, the water structure is even more disordered adjacent to the central groups (vertical dashed red line). The values of $P(0.76)$ relative to the respective bulk water value are plotted for each of the four segments of PEO-5 in Fig. 3e (unfilled data points) along with the average results from simulations of polyethers ranging from the monomer to a 35-mer (filled data points). As can be seen, the changes in interfacial water structure at specific sites along the polyether chains (Fig. 3e) correspond to the anion binding interactions (Fig. 2c,e). This idea is depicted schematically in Fig. $3 a$ as the ordered and disordered water structures.

Correlation of binding affinity and water structure. The adsorption of NaSCN to a specific position on the polyether chain reflects the degree of order in the hydration shell at that site. This relationship is quantified in Fig. 4 a, which plots the adsorption free energies, as measured by NMR (Fig. 2c), against the segment-specific ratios of the Raman $\mathrm{OH}$ stretch bands (Fig. 3c). An analogous correlation from the simulation studies is shown in Fig. $4 \mathrm{~b}$ to relate the normalized preferential interaction coefficient (Fig. 2e) to the probability of forming water networks with more tetrahedral order (Fig. 3e). The consequences of this relationship are apparent in 



Fig. 2 | The interaction of NaSCN with polyethers. a, Schematic illustration of SCN- binding to the centre of PEO-5, but not to its termini. b, Salt-induced ${ }^{1} \mathrm{H}$ NMR chemical shifts, $\Delta \delta$, are used to probe the interactions of $\mathrm{SCN}^{-}$with the central (red), penultimate (orange) and end (green) methylenes as well as terminal methyl groups (blue) of PEO-5. The solid lines are fits to equation (1). Error bars represent the standard deviation of two to three solution preparations and are smaller than most of the data points. c, The free energy of NaSCN adsorption, $\Delta G_{\text {ads, }}$ for the individual segments of PEO-5 (unfilled symbols) quantify the heterogeneous adsorption of $\mathrm{SCN}^{-}$to the chain. The average $\Delta G_{\text {ads }}$ values (filled symbols) for these segments are obtained from multiple PEOs $(1,2,3,4,5,2,000,20,000)$ and PEGs $(6,8,35,75,227)$. The error bars for PEO-5 are the standard deviation of two NaSCN titrations, and the error bars for the average values are obtained by error propagation calculations. The blue arrow denotes that $\Delta G_{\text {ads }}$ is weaker than the limit of detection at the chain's termini. The minimum and maximum estimates for the limit of detection are represented by the blue error bars. $\mathbf{d}$, Structures of various PEOs, illustrating the chain length dependence of the NaSCN binding site specificity. $\mathbf{e}$, The preferential interaction coefficient, $\mu_{23}$, for the individual segments of PEO-5 (unfilled symbols) and averages (filled symbols) are obtained from simulations for various PEOs $(1,3,5,7,14,35)$ and support the NMR results. Error bars are the standard error of the mean for each segment in the simulation. The NMR experiments, MD simulations and the role of cation and anion identity are described in Supplementary Sections 1 and 2 (Supplementary Figs. 1-40).

the ion density map shown in Fig. 4c. Hot spots for $\mathrm{SCN}^{-}$adsorption occur along the middle of the PEO-5 chain (yellow isobars), while $\mathrm{Na}^{+}$binding is localized to the ether oxygens (green isobars). Importantly, the $\mathrm{SCN}^{-}$binding sites match the water structure heat map in Fig. 4 d, which depicts the value of $P / P_{\text {bulk }}(q=0.76)$ for water molecules solvating specific heavy atoms along the chain. The heavy atoms are coloured blue where the value of $P / P_{\text {bulk }}$ is high, indicating an ordered solvation shell, and red where the value is low, denoting disordered hydration. Together, these results demonstrate that broken hydrogen bonds in the hydration shells at the polymer's centre are more readily displaced by weakly hydrated anions compared to the termini (Fig. 1). On the other hand, the more tetrahedrally ordered hydration shells of the monomer and the polymer's termini appear to inhibit the adsorption of $\mathrm{SCN}^{-}$.

\section{Discussion}

In this work, the concept of molecular surface curvature is employed to explain the correlation between interfacial water structure and the binding of Hofmeister anions. The systematic investigation of chain length effects on $\mathrm{SCN}^{-}$adsorption and water structure reveals distinct behaviours at the methyl termini and the internal methylene moieties along the chain. Location-specific analysis is consistent with the idea that the centre of the chain resembles a cylinder-like structure, while the termini are reminiscent of half spheres (Fig. 1). Water is more tetrahedrally ordered near the latter regions. By contrast, along the centre of the chain, there are weaker and less tetrahedral hydrogen bonds. It is at these sites of disordered hydrogen bonding where the strongest affinity for $\mathrm{SCN}^{-}$occurs. The correlation in Fig. 4 provides a systematic depiction of the ability for 

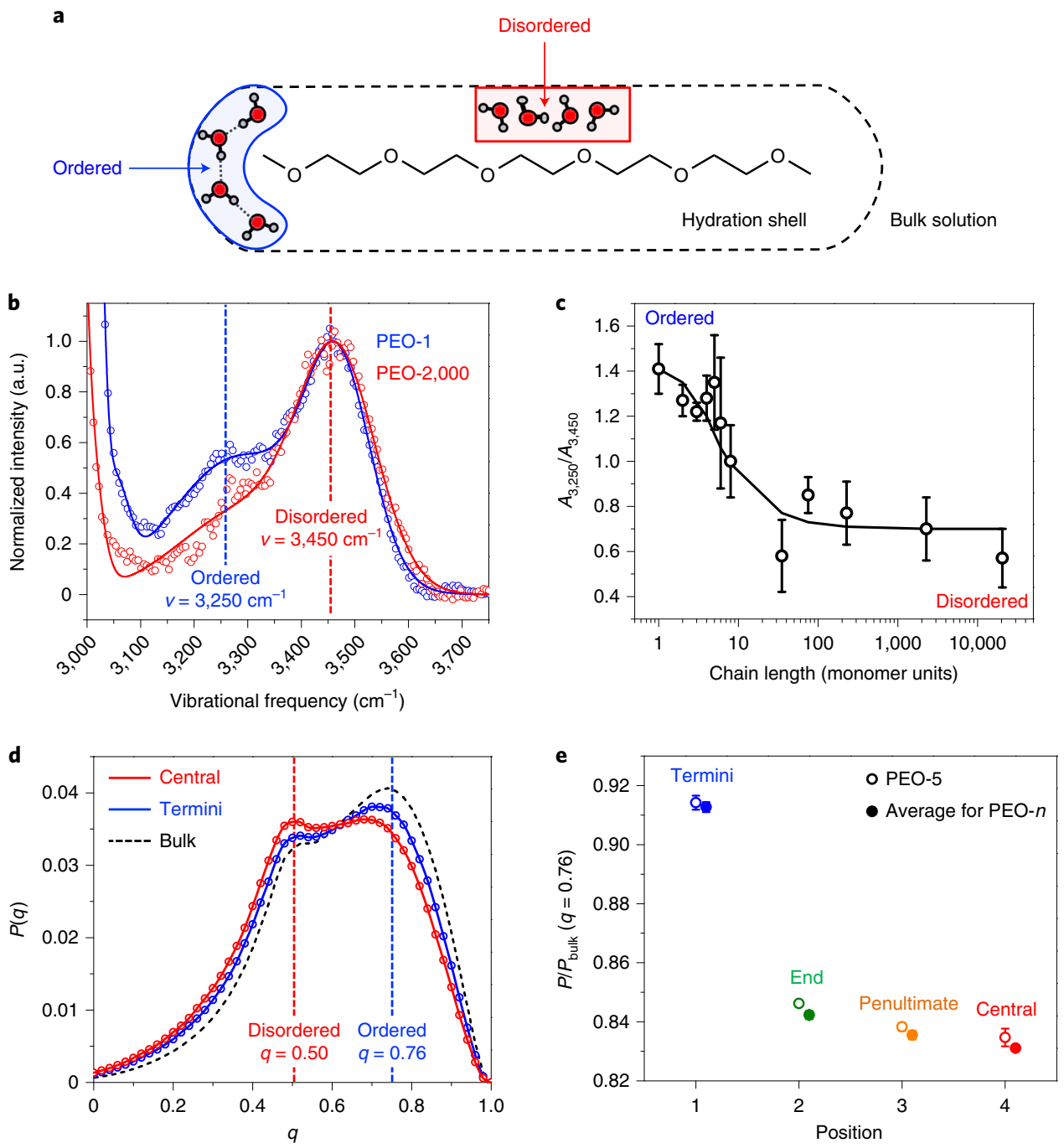

Fig. 3 | Structure of water in polyether hydration shells. a, Schematic illustration of PEO-5 hydration, where the hydrogen-bonded network of water is more ordered at the termini (blue region) than at the centre of the chain (red region). b. Hydration shell spectra of PEO-1 (blue) and PEO-2,000 (red) obtained by Raman spectroscopy and multivariate curve resolution analysis. The solid curves represent fits to the data using a sum of Gaussian peaks. As the chain length is increased, the intensity of the peak corresponding to tetrahedrally ordered water $\left(3,250 \mathrm{~cm}^{-1}\right.$, dashed blue line $)$ decreases relative to the disordered water peak $\left(3,450 \mathrm{~cm}^{-1}\right.$, dashed red line). $\mathbf{c}$, The area ratio of these two peaks, $A_{3,250} / A_{3,450}$, shows the chain length-induced structural transformation of the polyether hydration shell. Error bars are the standard deviation of three solution preparations. The solid curve is the best fit of the data to a segmented chain model, which quantifies the values of $A_{3,250} / A_{3,450}$ for each segment of the chain. $\mathbf{d}$, The probability distribution of the tetrahedral order parameter, $P(q)$, for bulk water (black dashed line) versus water hydrating the termini (blue data) and centre (red data) of PEO-5. Water molecules in tetrahedrally ordered hydrogen-bonding networks ( $q=0.76$, dashed blue line) are less populated at the centre of the chain than at the termini. e, The probability of observing tetrahedrally ordered water in the polyether hydration shell relative to the bulk solution, $P / P_{\text {buk }}(q=0.76)$, for each segment of PEO-5 (unfilled symbols). The average values (filled symbols) are obtained from simulations of the same solutes described in Fig. 2. Error bars are the standard error of the mean for each segment in the simulation. The Raman-MCR experiments and $q$ computations are described in Supplementary Section 3 (Supplementary Figs. 41-54).

water structure to completely reverse the nature of the interactions of weakly hydrated anions with nonpolar surfaces; that is, $\Delta G_{\text {ads }}$ varies from -5.3 to $>+2.2 \mathrm{~kJ} \mathrm{~mol}^{-1}\left(K_{\mathrm{D}}=0.1\right.$ to $\left.>2.4 \mathrm{M}\right)$.

The relationship between surface curvature and interfacial water structure is central to the hydrophobic effect. Small and convex aliphatic molecules are significantly more soluble than their larger and flatter counterparts, despite their chemical similarity ${ }^{39,40}$. Stillinger envisioned that bulk-like hydrogen bonding of water would be preserved around small solutes, but disrupted near the surfaces of large solutes, which more closely resemble the air/ water interface ${ }^{26}$. Quantitative theories and computer simulations predict a crossover between these two regimes at nanometre length scales $^{41,42}$. Simulations and spectroscopy experiments have observed signatures of this crossover in the dynamics of water at interfaces with varying curvature ${ }^{43-45}$. The hydration of $n$-alcohols as a function of chain length and the solvation free energy of polymers with differently sized aromatic side chains both exhibit signatures of the predicted crossover length scale ${ }^{28,46}$. The distance between the termini and end group methylenes of the polyethers investigated in this work is $\sim 0.5 \mathrm{~nm}$, which is very close to the characteristic crossover length for water structure and hydrophobicity ${ }^{27,28,42}$. As such, the internal segments of the chain behave like surfaces with reduced curvature, which create water structure defects that are readily displaced by weakly hydrated anions.

The chain length dependence and site specificity of anion adsorption and hydration structure is not limited to NaSCN and PEOs. 

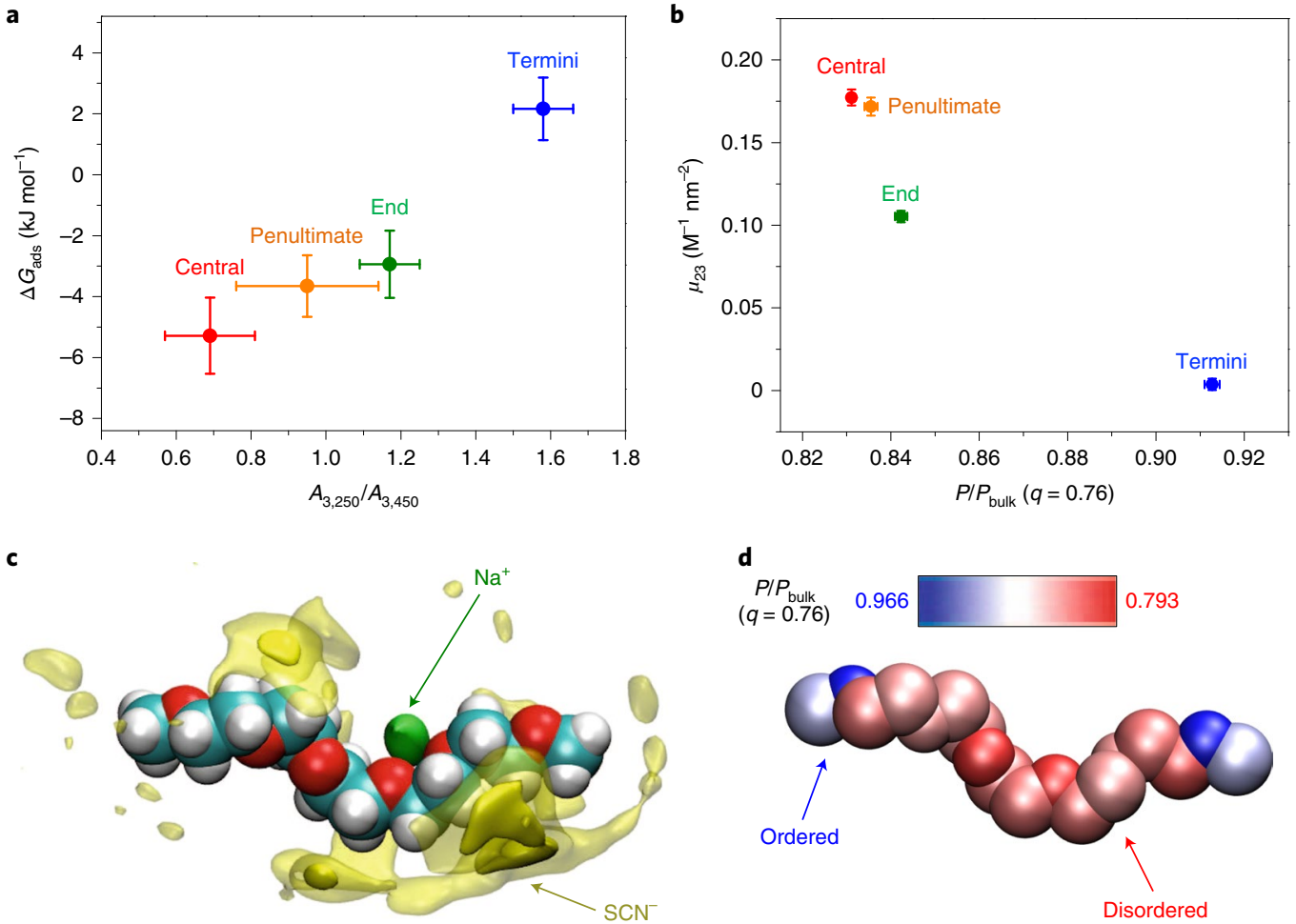

Fig. 4 | The role of interfacial water structure on NaSCN adsorption to polyether chains. a, Correlation of the free energy of NaSCN adsorption, $\Delta G_{\text {ads }}$ and the ratio of ordered to disordered water in the hydration shell, $A_{3,250} / A_{3,450}$. b. Correlation of the NaSCN preferential interaction coefficient, $\mu_{23}$, versus the probability of observing tetrahedrally ordered water in the hydration shell, $P / P_{\text {bulk }}(q=0.76)$. The data points in $\mathbf{a}$ and $\mathbf{b}$ represent the average values for each segment of the polyether chains described in Figs. 2 and 3. Error bars in $\mathbf{a}$ and $\mathbf{b}$ are the standard deviation and the standard error of the mean, respectively. The strong correlations observed in both the experiments and simulations suggest that the adsorption of NaSCN to the interior segments of the polyether chains is enhanced by the displacement of disordered water structures from the polyether hydration shell. c, The NaSCN density map shows the heterogeneous accumulation of ions around the PEO-5 chain. The opaque and transparent clouds represent isosurfaces where SCN- (yellow) or $\mathrm{Na}^{+}$ (green) is six or three times the bulk density, respectively. $\mathbf{d}$, The heat map of $P / P_{\text {bulk }}(q=0.76)$ values depicts the distinct tetrahedral structure of water at the centre and termini of the PEO- 5 hydration shell. Each heavy atom is coloured according to the provided scale. The correspondence of the $\mathrm{SCN}^{-}$ accumulation in $\mathbf{c}$ with the tetrahedral water structure in $\mathbf{d}$ provides a visual representation of the site-specific correlations in $\mathbf{a}$ and $\mathbf{b}$. Further discussion of the correlations is provided in Supplementary Section 4 (Supplementary Figs. 55 and 56).

As discussed in Supplementary Sections 2 and 5 (Supplementary Figs. 23-31 and 57-59), these effects apply to other weakly hydrated anions, like $\mathrm{I}^{-}$, and polymers, such as polyacrylamides. Moreover, the results are similar when other alkali metal cations are employed, like $\mathrm{Li}^{+}$and $\mathrm{Cs}^{+}$. Despite their chemical differences, this simple model invoking surface curvature predicts how anion-specific interactions vary with binding site location and chain length. The bifurcation in anion-hydrophobic interactions leads to divergent macroscopic behaviour for small molecules and polymers. Indeed, weakly hydrated anions reduce the solubility of small molecules in aqueous solutions, but inhibit the hydrophobic collapse of thermoresponsive polymers and denature proteins $s^{1,11,25,47}$. The incorporation of local surface curvature concepts into theoretical models should improve predictions for anion-specific effects on both small molecules and protein macromolecules. In a broader sense, the binding of anions as a function of molecular surface curvature should impact a variety of phenomena involving hydrophobic interfaces, ranging from the formation of methane clathrates to host-guest supramolecular complexation.

\section{Online content}

Any methods, additional references, Nature Research reporting summaries, source data, extended data, supplementary information, acknowledgements, peer review information; details of author contributions and competing interests; and statements of data and code availability are available at https://doi.org/10.1038/ s41557-021-00805-Z.

Received: 23 January 2021; Accepted: 31 August 2021; Published online: 1 November 2021

\section{References}

1. Bye, J. W. \& Falconer, R. J. Thermal stability of lysozyme as a function of ion concentration: a reappraisal of the relationship between the Hofmeister series and protein stability. Protein Sci. 22, 1563-1570 (2013).

2. Gibb, C. L. D. \& Gibb, B. C. Anion binding to hydrophobic concavity is central to the salting-in effects of Hofmeister chaotropes. J. Am. Chem. Soc. 133, 7344-7347 (2011).

3. Ray, A. \& Nemethy, G. Effects of ionic protein denaturants on micelle formation by nonionic detergents. J. Am. Chem. Soc. 93, 6787-6793 (1971).

4. Zhang, Y. \& Cremer, P. S. Interactions between macromolecules and ions: the Hofmeister series. Curr. Opin. Chem. Biol. 10, 658-663 (2006).

5. Petersen, P. B. \& Saykally, R. J. On the nature of ions at the liquid water surface. Annu. Rev. Phys. Chem. 57, 333-364 (2006).

6. Tobias, D. J. \& Hemminger, J. C. Getting specific about specific ion effects. Science 319, 1197-1198 (2008).

7. Pegram, L. M. \& Record, M. T. Thermodynamic origin of Hofmeister ion effects. J. Phys. Chem. B 112, 9428-9436 (2008).

8. Zhang, Y. \& Cremer, P. S. Chemistry of Hofmeister anions and osmolytes. Annu. Rev. Phys. Chem. 61, 63-83 (2010).

9. Lo Nostro, P. \& Ninham, B. W. Hofmeister phenomena: an update on ion specificity in biology. Chem. Rev. 112, 2286-2322 (2012). 
10. Okur, H. I. et al. Beyond the Hofmeister series: ion-specific effects on proteins and their biological functions. J. Phys. Chem. B 121, 1997-2014 (2017).

11. Zhang, Y., Furyk, S., Bergbreiter, D. E. \& Cremer, P. S. Specific ion effects on the water solubility of macromolecules: PNIPAM and the Hofmeister series. J. Am. Chem. Soc. 127, 14505-14510 (2005).

12. Cho, Y. et al. Effects of Hofmeister anions on the phase transition temperature of elastin-like polypeptides. J. Phys. Chem. B 112, 13765-13771 (2008).

13. Rembert, K. B. et al. Molecular mechanisms of ion-specific effects on proteins. J. Am. Chem. Soc. 134, 10039-10046 (2012).

14. Rembert, K. B., Okur, H. I., Hilty, C. \& Cremer, P. S. An NH moiety is not required for anion binding to amides in aqueous solution. Langmuir 31 3459-3464 (2015).

15. Dang, L. X. Computational study of ion binding to the liquid interface of water. J. Phys. Chem. B 106, 10388-10394 (2002).

16. Jungwirth, P. \& Tobias, D. J. Ions at the air/water interface. J. Phys. Chem. B 106, 6361-6373 (2002).

17. Petersen, P. B., Saykally, R. J., Mucha, M. \& Jungwirth, P. Enhanced concentration of polarizable anions at the liquid water surface: SHG spectroscopy and MD simulations of sodium thiocyanide. J. Phys. Chem. B 109, 10915-10921 (2005).

18. Otten, D. E., Shaffer, P. R., Geissler, P. L. \& Saykally, R. J. Elucidating the mechanism of selective ion adsorption to the liquid water surface. Proc. Natl Acad. Sci. USA 109, 701-705 (2012).

19. Fox, J. M. et al. Interactions between Hofmeister anions and the binding pocket of a protein. J. Am. Chem. Soc. 137, 3859-3866 (2015).

20. McCaffrey, D. L. et al. Mechanism of ion adsorption to aqueous interfaces: graphene/water vs. air/water. Proc. Natl Acad. Sci. USA 114, 13369-13373 (2017).

21. Sokkalingam, P., Shraberg, J., Rick, S. W. \& Gibb, B. C. Binding hydrated anions with hydrophobic pockets. J. Am. Chem. Soc. 138, 48-51 (2016).

22. Sullivan, M. R., Yao, W., Tang, D., Ashbaugh, H. S. \& Gibb, B. C. The thermodynamics of anion complexation to nonpolar pockets. J. Phys. Chem. B 122, 1702-1713 (2018).

23. Rankin, B. M. \& Ben-Amotz, D. Expulsion of ions from hydrophobic hydration shells. J. Am. Chem. Soc. 135, 8818-8821 (2013).

24. Balos, V., Kim, H., Bonn, M. \& Hunger, J. Dissecting Hofmeister effects: direct anion-amide interactions are weaker than cation-amide binding. Angew. Chem. Int. Ed. 55, 8125-8128 (2016).

25. Long, F. A. \& McDevit, W. F. Activity coefficients of nonelectrolyte solutes in aqueous salt solutions. Chem. Rev. 51, 119-169 (1952).

26. Stillinger, F. H. Structure in aqueous solutions of nonpolar solutes from the standpoint of scaled-particle theory. J. Solution Chem. 2, 141-158 (1973).

27. Chandler, D. Interfaces and the driving force of hydrophobic assembly. Nature 437, 640-647 (2005)

28. Davis, J. G., Gierszal, K. P., Wang, P. \& Ben-Amotz, D. Water structural transformation at molecular hydrophobic interfaces. Nature 491, 582-585 (2012).

29. Hande, V. R. \& Chakrabarty, S. Structural order of water molecules around hydrophobic solutes: length-scale dependence and solute-solvent coupling. J. Phys. Chem. B 119, 11346-11357 (2015).
30. Pierce, V., Kang, M., Aburi, M., Weerasinghe, S. \& Smith, P. E. Recent applications of Kirkwood-Buff theory to biological systems. Cell Biochem. Biophys. 50, 1-22 (2008).

31. Knowles, D. B. et al. Chemical interactions of polyethylene glycols (PEGs) and glycerol with protein functional groups: applications to effects of PEG and glycerol on protein processes. Biochemistry 54, 3528-3542 (2015).

32. Fega, K. R., Wilcox, A. S. \& Ben-Amotz, D. Application of Raman multivariate curve resolution to solvation-shell spectroscopy. Appl. Spectrosc. 66, 282-288 (2012).

33. Walrafen, G. E., Fisher, M. R., Hokmabadi, M. S. \& Yang, W. -H. Temperature dependence of the low- and high-frequency Raman scattering from liquid water. J. Chem. Phys. 85, 6970-6982 (1986).

34. D’Arrigo, G., Maisano, G., Mallamace, F., Migliardo, P. \& Wanderlingh, F. Raman scattering and structure of normal and supercooled water. J. Chem. Phys. 75, 4264-4270 (1981).

35. Sun, Q. Local statistical interpretation for water structure. Chem. Phys. Lett. 568-569, 90-94 (2013).

36. Harada, Y. et al. Probing the $\mathrm{OH}$ stretch in different local environments in liquid water. J. Phys. Chem. Lett. 8, 5487-5491 (2017).

37. Morawietz, T. et al. The interplay of structure and dynamics in the Raman spectrum of liquid water over the full frequency and temperature range. $J$. Phys. Chem. Lett. 9, 851-857 (2018).

38. Duboué-Dijon, E. \& Laage, D. Characterization of the local structure in liquid water by various order parameters. J. Phys. Chem. B 119, 8406-8418 (2015).

39. Mackay, D. \& Shiu, W. Y. A critical review of Henry's Law constants for chemicals of environmental interest. J. Phys. Chem. Ref. Data 10, 1175-1199 (1981)

40. Meyer, D. E. \& Chilkoti, A. Quantification of the effects of chain length and concentration on the thermal behavior of elastin-like polypeptides. Biomacromolecules 5, 846-851 (2004).

41. Lee, C., McCammon, J. A. \& Rossky, P. J. The structure of liquid water at an extended hydrophobic surface. J. Chem. Phys. 80, 4448-4455 (1984).

42. Lum, K., Chandler, D. \& Weeks, J. D. Hydrophobicity at small and large length scales. J. Phys. Chem. B 103, 4570-4577 (1999).

43. Laage, D., Stirnemann, G. \& Hynes, J. T. Why water reorientation slows without iceberg formation around hydrophobic solutes. J. Phys. Chem. B 113, 2428-2435 (2009).

44. Petersen, C., Tielrooij, K.-J. \& Bakker, H. J. Strong temperature dependence of water reorientation in hydrophobic hydration shells. J. Chem. Phys. 130, 214511 (2009)

45. Xi, E. et al. Hydrophobicity of proteins and nanostructured solutes is governed by topographical and chemical context. Proc. Natl Acad. Sci. USA 114, 13345-13350 (2017).

46. Li, I. T. S. \& Walker, G. C. Signature of hydrophobic hydration in a single polymer. Proc. Natl Acad. Sci. USA 108, 16527-16532 (2011).

47. von Hippel, P. H. \& Wong, K.-Y. On the conformational stability of globular proteins. The effects of various electrolytes and nonelectrolytes on the thermal ribonuclease transition. J. Biol. Chem. 240, 3909-3923 (1965).

Publisher's note Springer Nature remains neutral with regard to jurisdictional claims in published maps and institutional affiliations.

(c) The Author(s), under exclusive licence to Springer Nature Limited 2021 


\section{Methods}

Reagents. High-purity sodium thiocyanate was used in all experiments (NaSCN, 99.99\%). The monomer and oligomers of polyethylene glycols (PEG- $n$ ) were liquids and at least 95\% pure: ethylene glycol (PEG-1, 99.8\%), diethylene glycol (PEG-2, $\geq 99.0 \%$,), triethylene glycol (PEG-3, 99\%), tetraethylene glycol (PEG-4, 99\%), pentaethylene glycol (PEG-5, 98\%), hexaethylene glycol (PEG-6 $97 \%$ ) and octaethylene glycol (PEG-8, $\geq 95 \%$ ). The monomer and oligomers of PEO (PEO- $n$ ) were also liquids with at least $99 \%$ purity: 1,2-dimethoxyethane (PEO-1, 99.9\%), diethylene glycol dimethyl ether (PEO-2, 99.5\%), triethylene glycol dimethyl ether (PEO-3, 99\%), tetraethylene glycol dimethyl ether (PEO-4, $\geq 99 \%$ ) and polyethylene glycol dimethyl ether $M_{\mathrm{n}} \approx 250 \mathrm{~g} \mathrm{~mol}^{-1}(\mathrm{PEO}-5, \mathrm{n} 20 / \mathrm{D}$ $1.441)$, where $M_{\mathrm{n}}$ is the number average molecular weight. The longer-chain PEGs and PEOs were solid powders: polyethylene glycol $M_{\mathrm{w}} \approx 1,500 \mathrm{~g} \mathrm{~mol}^{-1}$ (PEG-35), polyethylene glycol $M_{\mathrm{w}} \approx 3,350 \mathrm{~g} \mathrm{~mol}^{-1}$ (PEG-75), polyethylene glycol $M_{\mathrm{w}} \approx 10,000 \mathrm{~g} \mathrm{~mol}^{-1}$ (PEG-227), polyethylene oxide $M_{\mathrm{v}} \approx 100,000 \mathrm{~g} \mathrm{~mol}^{-1}$ (PEO-2,000) and polyethylene oxide $M_{\mathrm{v}} \approx 900,000 \mathrm{~g} \mathrm{~mol}^{-1}$ (PEO-20,000), where $M_{\mathrm{w}}$ and $M_{\mathrm{v}}$ are the weight average and the viscosity average molecular weights, respectively.

Sample preparation. $\mathrm{NaSCN}$ was dried at $115^{\circ} \mathrm{C}$ for 8 hours before preparing solutions to remove adsorbed water. All solutions were prepared using $18 \mathrm{M} \Omega \mathrm{cm}$ deionized water. The stock salt solutions were prepared gravimetrically in volumetric flasks. The stock polyether solutions for the liquid polyethers were prepared volumetrically by diluting a volume of the pure liquid with water. Stock polyether solutions of the solid PEGs were prepared gravimetrically. The salt and polyether stock solutions were mixed volumetrically with water to prepare the ternary solutions. The final monomer concentration was $0.25 \mathrm{M}\left(\sim 11 \mathrm{mg} \mathrm{ml}^{-1}\right)$ for all chain lengths. The volumes were assumed to be additive.

NMR spectroscopy. ${ }^{1} \mathrm{H}$ NMR experiments were performed with precision coaxial insert NMR tubes. The external reference, $2 \mathrm{mg} \mathrm{ml}^{-1}$ 4,4-dimethyl-4-silapentane1 -sulfonic acid, and the locking agent, deuterium oxide, were loaded into a coaxial insert. The insert was then positioned in the centre of a $5-\mathrm{mm}$ precision sample tube. The spectra were collected on a $500-\mathrm{MHz}$ spectrometer. After the samples were equilibrated to $298 \mathrm{~K}$ for $2 \mathrm{~min}$, one-dimensional ${ }^{1} \mathrm{H}$ spectra were acquired using Bruker's standard excitation sculpting for water suppression ${ }^{48}$. Additional details for the NMR instrumentation, pulse sequence and data analysis are described in the Supplementary Information.

Raman spectroscopy. Raman spectra were collected on a home-built instrument. The 514.5-nm line of a mixed Ar-Kr gas laser was focused to the centre of a 1-cm quartz cuvette. The unpolarized Raman signal was collected in a backscattering geometry and directed to a spectrometer. The power of the incident light at the sample was adjusted to $50 \mathrm{~mW}$ and the collection time was $5 \mathrm{~min}$. The solutions contained $0.5 \mathrm{M}$ monomer concentration $\left(\sim 22 \mathrm{mg} \mathrm{ml}^{-1}\right)$ for all chain lengths. Hydration shell spectra were extracted using a multivariate curve resolution (MCR) algorithm written for IGOR Pro by D. Ben-Amotz at Purdue University. The inputs for the MCR analysis were a spectrum of an aqueous solution containing a polyether and a spectrum of pure water. The output was the deconvolution of the solution spectrum into components for pure water and another for the hydration shell. An extended discussion of the spectrometer design and MCR analysis is provided in the Supplementary Information.

Simulation composition. All-atom molecular dynamics (MD) studies were performed with polyether chains that were $1,3,5,7,14$ or 35 monomer units long $^{49,50}$. Each simulation involved 3,100-3,400 water molecules that were parameterized with the extended simple point charge (SPC/E) force field and the number of polymer chains was adjusted to mimic the constant monomer concentration employed in the experiment ${ }^{51}$. All simulation boxes reached an approximate volume of $4.7 \times 4.7 \times 4.7 \mathrm{~nm}^{3}$ after an initial $N P T$ equilibration (20 ns). The evaluation of water structure was conducted on MD simulations containing only one polymer chain to avoid the potential bias from chain-chain interactions. The ion-polymer interactions were evaluated from simulations of a $1 \mathrm{M}$ salt solution that were parameterized using non-polarizable force fields for the ions $s^{13,52,53}$. The polymers were flexible in all of the simulations, except simulations that were performed to create the three-dimensional density maps, where the polymer was constrained to the average conformation.

Simulation algorithms and data analysis. Simulations were carried out over several hundred nanoseconds with a time step of $2 \mathrm{fs}$ with the GROMACS simulation package ${ }^{54}$. Trajectories were stored at 1 -ps intervals, resulting in $10^{5}$ to $10^{6}$ samples for subsequent data analysis. The systems were constrained to $300 \mathrm{~K}$ and $1 \mathrm{~atm}$ using a velocity-rescale for a canonical sampling thermostat and a Parrinello-Rahman barostat with coupling constants of 0.1 and 2 ps, respectively ${ }^{55,56}$. Three-dimensional periodic boundary conditions were applied. Long-range electrostatic interactions beyond the non-bonded cutoff of $10 \AA$ were accounted for using the particle mesh Ewald method on a $0.16-\mathrm{nm}_{\text {grid }}{ }^{57}$. All bonds containing hydrogen atoms were constrained using the LINCS algorithm ${ }^{58}$ Preferential binding of ions to the polyether surfaces was calculated by means of
Kirkwood-Buff theory ${ }^{30,59}$. Water structure was evaluated using the tetrahedral order parameter ${ }^{38,60}$.

\section{Data availability}

The datasets generated during and/or analysed during the current study are available via the following DOIs: Fig. 2, https://doi.org/10.5281/zenodo.5123016; Fig. 3, https://doi.org/10.5281/zenodo.5123100; Fig. 4, https://doi.org/10.5281/ zenodo.5123104. Source data are provided with this paper. Source data for the Supplementary figures are available at https://doi.org/10.5281/zenodo.5123295.

\section{Code availability}

The codes and algorithms generated during the current study are available from the corresponding author on reasonable request.

\section{References}

48. Hwang, T. L. \& Shaka, A. J. Water suppression that works. Excitation sculpting using arbitrary wave-forms and pulsed-field gradients. J. Magn. Reson. A 112, 275-279 (1995).

49. Lee, H., Venable, R. M., MacKerell, A. D. \& Pastor, R. W. Molecular dynamics studies of polyethylene oxide and polyethylene glycol: hydrodynamic radius and shape anisotropy. Biophys. J. 95, 1590-1599 (2008).

50. Chudoba, R., Heyda, J. \& Dzubiella, J. Temperature-dependent implicit-solvent model of polyethylene glycol in aqueous solution. J. Chem. Theory Comput. 13, 6317-6327 (2017).

51. Berendsen, H. J. C., Grigera, J. R. \& Straatsma, T. P. The missing term in effective pair potentials. J. Phys. Chem. 91, 6269-6271 (1987).

52. Heyda, J., Vincent, J. C., Tobias, D. J., Dzubiella, J. \& Jungwirth, P. Ion specificity at the peptide bond: molecular dynamics simulations of $\mathrm{N}$-methylacetamide in aqueous salt solutions. J. Phys. Chem. B 114, 1213-1220 (2010).

53. Kř́žžek, T. et al. Electrophoretic mobilities of neutral analytes and electroosmotic flow markers in aqueous solutions of Hofmeister salts. Electrophoresis 35, 617-624 (2014).

54. Abraham, M. J. et al. GROMACS: high performance molecular simulations through multi-level parallelism from laptops to supercomputers. SoftwareX 1-2, 19-25 (2015).

55. Bussi, G., Donadio, D. \& Parrinello, M. Canonical sampling through velocity rescaling. J. Chem. Phys. 126, 14101 (2007).

56. Parrinello, M. \& Rahman, A. Polymorphic transitions in single crystals: a new molecular dynamics method. J. Appl. Phys. 52, 7182-7190 (1981).

57. Essmann, U. et al. A smooth particle mesh Ewald method. J. Chem. Phys. 103, 8577-8593 (1995).

58. Hess, B. P-LINCS: a parallel linear constraint solver for molecular simulation. J. Chem. Theory Comput. 4, 116-122 (2008).

59. Paterová, J. et al. Reversal of the Hofmeister series: specific ion effects on peptides. J. Phys. Chem. B 117, 8150-8158 (2013).

60. Errington, J. R. \& Debenedetti, P. G. Relationship between structural order and the anomalies of liquid water. Nature 409, 318-321 (2001).

\section{Acknowledgements}

We thank C. Chen, Z. Tian and H. Allcock for poly- $N, N$-diethylacrylamide (PDEA) synthesis (The Pennsylvania State University), T. Mal and C. Pacheco for NMR assistance, as well as D. Ben-Amotz and W. Noid for insightful discussions. P.S.C. thanks the National Science Foundation (CHE-2004050) for funding support. J.H. acknowledges support from the Czech Science Foundation (grant no. 20-24155 S) and the Ministry of Education, Youth and Sports of the Czech Republic through the e-INFRA CZ (ID: 90140).

\section{Author contributions}

The project and mechanism were conceptualized by B.A.R., H.I.O. and P.S.C. The work was designed and the methods were developed with the help of all authors. Experimental data were acquired and analysed by B.A.R., H.I.O. and C.Y., while J.H. ran, analysed and wrote software algorithms for the computer simulations. B.A.R., H.I.O., C.Y., J.H. and P.S.C. interpreted the data. The original draft was written by B.A.R., H.I.O. and P.S.C. The manuscript was revised and edited by B.A.R., H.I.O, J.H. and P.S.C.

\section{Competing interests}

The authors declare no competing interests.

\section{Additional information}

Supplementary information The online version contains supplementary material available at https://doi.org/10.1038/s41557-021-00805-z.

Correspondence and requests for materials should be addressed to Paul S. Cremer.

Peer review information Nature Chemistry thanks Amish Patel and the other, anonymous, reviewer(s) for their contribution to the peer review of this work.

Reprints and permissions information is available at www.nature.com/reprints. 